EPJ Web of Conferences 66, 03035 (2014)

DOI: $10.1051 /$ epjconf/ 20146603035

(C) Owned by the authors, published by EDP Sciences, 2014

\title{
Pseudo-critical clusterization in nuclear multifragmentation
}

\author{
D. Gruyer ${ }^{1, a}$, J.D. Frankland ${ }^{1}$, R. Botet ${ }^{2}$, M. Płoszajczak ${ }^{1}$, E. Bonnet ${ }^{1}$, A. Chbihi ${ }^{1}$, and P. Marini ${ }^{1}$ \\ for the INDRA collaboration \\ ${ }^{1}$ GANIL, CEA-DSM/CNRS-IN2P3, Bvd. Henri Becquerel, F-14076 Caen Cedex, France \\ ${ }^{2}$ Laboratoire de Physique du Solide, Université de Paris-Sud, F-91405 Orsay, France
}

\begin{abstract}
In this contribution we show that the biggest fragment charge distribution in central collisions of ${ }^{129} \mathrm{Xe}+{ }^{n a t} \mathrm{Sn}$ leading to multifragmentation is an admixture of two asymptotic distributions observed for the lowest and highest bombarding energies. The evolution of the relative weights of the two components with bombarding energy is shown to be analogous to that observed as a function of time for the largest cluster produced in irreversible aggregation for a finite system. We infer that the size distribution of the largest fragment in nuclear multifragmentation is also characteristic of the time scale of the process, which is largely determined by the onset of radial expansion in this energy range.
\end{abstract}

Phase transitions play a central role in many fields of physics. Indeed, they allow us to investigate the equation of state and phase diagram of the system under study. The case of nuclear multifragmentation, as observed in intermediate energy heavy-ion collisions [1, 2], provides a unique opportunity to study not only thermodynamical properties of nuclear matter, but also phase transitions in finite systems.

In an infinite system, fluctuations are generally irrelevant and phase transitions can be characterized by the correlations between the control parameter and the order parameter (fig. 1(a)). With decreasing size of the system, fluctuations become more important and correlations are distorted (fig. 1(b)), such that for small system sizes (comparable to that of a nucleus), we are not able to identify the transition from this simple picture (fig. 1(c)). The universal character of order parameter fluctuations in finite systems [3] provides a good framework in which to address such questions. Within such a framework, it was shown that the size (atomic number) of the largest fragment produced in multifragmentation events, $\mathrm{Z}_{\max }$, behaves like an order parameter, i.e. the scaling properties of its fluctuations change with increasing energy [4]. This means that we know to which class of generic cluster models nuclear multifragmentation belongs, answering the question raised in Ref. [5]: it is an aggregation phenomenon ("condensation of vapor"), not a fragmentation process ("shattering of glass"). In ref. [6] it was shown that a distinct asymptotic form of the $\mathrm{Z}_{\max }$ distribution can be associated with each scaling regime: a quasi-Gaussian distribution at the lower bombarding energies $\left(\mathrm{E}_{\text {beam }} \lesssim 30 \mathrm{MeV} / \mathrm{A}\right)$, and a Gumbel distribution in the higher-energy disordered regime $\left(\mathrm{E}_{\text {beam }} \gtrsim 40 \mathrm{MeV} / \mathrm{A}\right)$.

Here we study in more detail the transition from one energy regime to the other, using new data on $\mathrm{Z}_{\max }$ distributions for ${ }^{129} \mathrm{Xe}+{ }^{\text {nat }} \mathrm{Sn}$ central collisions measured with INDRA [6, 7], at eight beam energies, between 25 and $50 \mathrm{MeV} / \mathrm{A}$. Within the hypothesis that at intermediate energies between the

ae-mail: diego.gruyer@ganil.fr

This is an Open Access article distributed under the terms of the Creative Commons Attribution License 2.0, which permits unrestricted use, distribution, and reproduction in any medium, provided the original work is properly cited. 


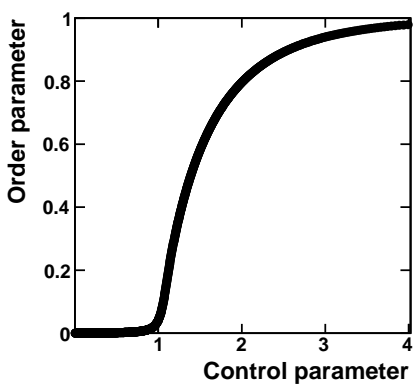

(a) $N \sim 10^{4}$ particles

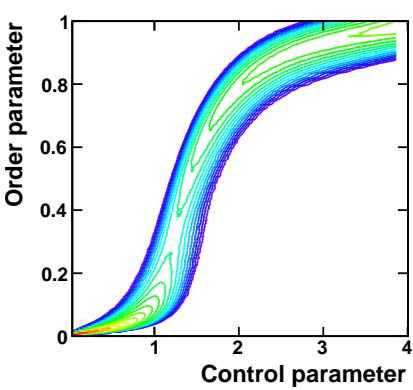

(b) $N \sim 200$ particles

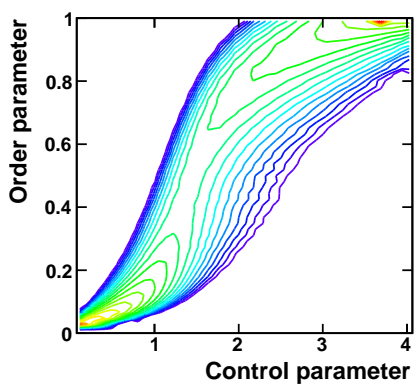

(c) $N \sim 50$ particles

Figure 1. (color online) Correlations between the order parameter and the control parameter for a critical phase transition occurring in systems of different sizes.

two regimes, $\mathrm{Z}_{\max }$ distributions can be described by an admixture of the two asymptotic forms [8], we fit them with the following function :

$$
f\left(\mathrm{Z}_{\max }\right)=\eta f_{G a}\left(\mathrm{Z}_{\max }\right)+(1-\eta) f_{G u}\left(\mathrm{Z}_{\max }\right),
$$

where, $f_{G a}$ and $f_{G u}$ are the Gaussian and the Gumbel component.

The results of the fits using eq. (1) are shown in fig. 2. It can be seen that, for all analyzed energies, experimental data are well reproduced by $f\left(\mathrm{Z}_{\max }\right)$. At the highest considered energy (50 $\mathrm{MeV} / \mathrm{A}), \mathrm{P}\left(\mathrm{Z}_{\max }\right)$ is an almost pure Gumbel distribution (fig. 2(d)). For lower bombarding energies (fig. 2(a-c)), both Gaussian and Gumbel contributions are present, and the relative importance of the Gaussian component increases with decreasing energy. In fig. 2(e) we present the evolution of the relative weight between the two components as a function of the beam energy. We observe not only two asymptotic regimes, but a continuous and smooth evolution between them.
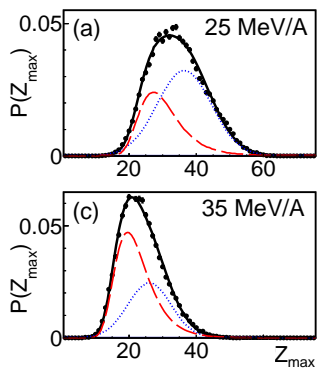
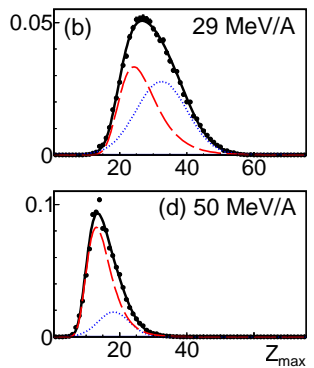

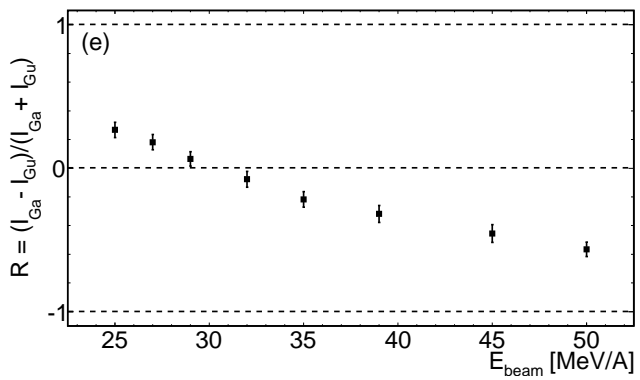

Figure 2. (Color online) Analysis of ${ }^{129} \mathrm{Xe}+{ }^{n a t} \mathrm{Sn}$ experimental data: (a-d) largest fragment charge distributions, (Black solid curve) best fit to the data using eq. (1), (Red dashed curve) Gumbel component, (Blue dotted curve) Gaussian component; and (e) evolution of the relative weight between the two components, as a function of the beam energy.

To interpret such evolution, we consider the Smoluchowski irreversible aggregation model, which describes an out-of-equilibrium clusterization process. It exhibits a second-order phase transition 
after a critical time $\left(t_{c}\right)$, whose order parameter is also the average size of the largest cluster, $s_{\max }$. The order parameter distribution for irreversible aggregation is not known exactly. For short time evolution $\left(t \ll t_{c}\right)$, there are only few aggregation events. So $s_{\max }$ is the largest of randomly distributed cluster sizes. $s_{\max }$ is then of extremal nature, and it results in a Gumbel distribution (fig. 3(a)). In this model, the average size of the largest cluster increases with time as more and more coalescence of smaller clusters takes place. At long times $\left(t \gg t_{c}\right)$, the order parameter is then essentially of additive nature. From the central limit theorem, this results in an asymptotic Gaussian distribution (fig. 3(d)). In the critical domain, finite size fluctuations are so large that similarly prepared systems can exhibit one or the other behavior. In this domain, the $s_{\max }$ distribution is an admixture of the two asymptotic forms (fig. 3(b-c)), with a continuous evolution of their relative weight (fig. 3(e)).
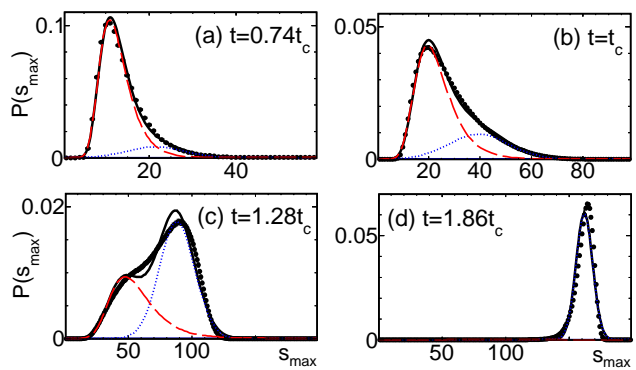

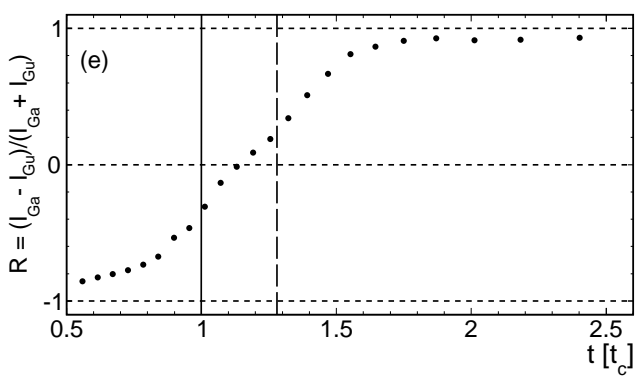

Figure 3. (Color online) Analysis of Smoluchowski calculations for $N=216$ particles: (a-d) largest cluster size distributions, (Black solid curve) best fit to the data using eq. (2), (Red dashed curve) Gumbel component, (Blue dotted curve) Gaussian component; (e) time evolution of the relative weight between the two components (eq. (3)), (vertical dashed line) pseudo-critical time $t_{c}^{*}$.

A strong similarity can be seen between the results of the analysis of irreversible aggregation calculations (fig. 3) and experimental data on ${ }^{129} \mathrm{Xe}+{ }^{\text {nat }}$ Sn central collisions (fig. 2). In the aggregation model the order parameter distribution depends on the time during which clusters can form. In central heavy-ion collisions, a determining factor for the time-scale of fragment formation is the radial expansion of the multifragmenting system, which increases with the bombarding energy [1]. Fragment sizes evolve as long as exchanges of nucleons take place between them, i.e. until the freeze-out condition is reached. It has been shown that, for central ${ }^{129} \mathrm{Xe}+{ }^{n a t} \mathrm{Sn}$ reactions, the onset of significant radial expansion occurs for beam energies above $25 \mathrm{MeV} / \mathrm{A}$ [7]. Therefore, the similarity between figs. 3 and 2 can be interpreted in terms of fragment size distributions being determined on shorter and shorter time-scales due to increasingly rapid expansion.

We have shown that the largest fragment size distribution in multifragmentation events is an admixture of the two asymptotic forms. A similar decomposition is observed in critical aggregation, indicating that the critical domain lies around $\mathrm{E}_{\text {beam }} \approx 30 \mathrm{MeV} / \mathrm{A}$ for the ${ }^{129} \mathrm{Xe}+{ }^{\text {nat }} \mathrm{Sn}$ system. We interpret such criticality as the onset of an "explosive" multifragmentation regime.

\section{Acknowledgements}

The authors would like to thank the staff of the GANIL Accelerator facility for their continued support during the experiments. D. G. gratefully acknowledges the financial support of the Commissariat à l'énergie Atomique and the Conseil Régional de Basse-Normandie. 


\section{References}

[1] B. Borderie, M.F. Rivet, Progress in Particle and Nuclear Physics 61, 551 (2008)

[2] WCI (World Consensus Initiative) Topical Volume: "Dynamics and Thermodynamics with Nuclear Degrees of Freedom", European Physics Journal A 30, 1 (2006)

[3] R. Botet, M. Ploszajczak, Physical Review E 62, 1825 (2000)

[4] R. Botet, M. Ploszajczak, A. Chbihi, B. Borderie, D. Durand and J.D. Frankland, Physical Review Letters 86, 3514 (2001)

[5] J. Aichelin, J. Huefner, Physics Letters B 136, 15 (1984)

[6] J.D. Frankland, A. Chbihi, A. Mignon, M.L.B. Blaich, R. Bittiger, B. Borderie, R. Bougault, J.L. Charvet, D. Cussol, R. Dayras et al., Physical Review C 71, 034607 (2005)

[7] E. Bonnet, B. Borderie, N. Le Neindre, M.F. Rivet, R. Bougault, A. Chbihi, R. Dayras, J.D. Frankland, E. Galichet, F. Gagnon-Moisan et al., Nuclear Physics A 816, 1 (2009)

[8] D. Gruyer, J.D. Frankland, R. Botet, M. Ploszajczak, E. Bonnet, A. Chbihi, G. Ademard, M. Boisjoli, B. Borderie, R. Bougault et al., Physical Review Letters 110, 172701+ (2013) 